\title{
Um Algoritmo Baseado em Aritmética Intervalar para Cálculo de Fluxo de Carga em Redes De Distribuição Considerando Incertezas
}

\author{
Helton Nascimento Alves'. Camila Nonato Alves². \\ ${ }^{1}$ Departamento de eletroeletrônica (DEE), Instituto Federal do Maranhão, São Luís, MA 65030-000,BRAZIL \\ (98)988298192 e-mail: helton@ifma.edu.br. \\ ${ }^{2}$ Engenharia Elétrica, Instituto Federal do Maranhão, São Luís, MA,BRAZIL (e-mail: \\ camilan@acad.ifma.edu.br)
}

\begin{abstract}
Traditional load flow methods are deterministic, i.e., all of their parameters are modeled for a given set of load, generation, and line data values. The solution of these methods distances itself from the real state of the electrical system, considering that variations in line data, uncertainties in loads and generation are inherent in the electric grid. This work presents an algorithm for the study of the load flow in distribution networks considering uncertainties in its parameters based on interval arithmetic. This technique is incorporated into the equations of the backward/forward sweep power flow summation method to transform it into a non-deterministic power flow. The results obtained by the proposed algorithm and the Monte Carlo simulation in four radial distribution systems are compared.
\end{abstract}

Resumo: Métodos tradicionais de fluxo de cargas são determinísticos, i.e., todos os seus parâmetros são modelados para um determinado conjunto de valores de geração, carga e dados de linha. A solução desses métodos distancia-se do real estado do sistema elétrico, visto que variações nos dados de linhas, incertezas nas cargas e geração são inerentes a rede elétrica. Esse trabalho apresenta um algoritmo para o estudo do fluxo de carga em redes de distribuição considerando incertezas em seus parâmetros com base na aritmética intervalar. Esta técnica é incorporada as equações do método de soma de potência para transformá-lo em um fluxo de carga não determinístico. Os resultados obtidos aplicando-se o algoritmo proposto e a Simulação de Monte Carlo em quatro alimentadores radiais são comparados.

Keywords: Backward/forward power summation method; Distribution networks; Interval arithmetic; Uncertainties; Monte Carlo Simulator.

Palavras-chaves: Método de soma de potência; Redes de distribuição; Aritmética intervalar; Incertezas; Simulação de Monte Carlo.

\section{INTRODUÇÃO}

No cálculo do fluxo de carga baseado em dados determinísticos, várias suposições são feitas distanciando os modelos aplicados do sistema real. Dessa forma, os fluxos e tensões calculados não representam com precisão os reais valores. Ao se considerar as incertezas dos dados de entrada do fluxo de carga, obtêm-se soluções mais realistas, permitindo que o tomador de decisão compreenda melhor as implicações de qualquer projeto ou decisão operacional $(\mathrm{Li}$, 2005).

Para tratar esse problema várias metodologias têm sido propostas baseadas principalmente em três vertentes: modelagem probabilística (Borkowska, 1974; Su, 2005), métodos baseados na teoria dos conjuntos fuzzy (Kenarangui \& Seifi, 1994; Bijwe et al, 2005) e aritmética intervalar (Pereira et al, 2012; Zhang et al, 2017; X. Liao et al 2017). Em especial, a aritmética intervalar trabalha com intervalos numéricos que aproximam dados incertos, sendo uma maneira simples de tratar as incertezas inerentes aos sistemas de energia elétrica, sendo usada como auxílio e ferramenta de pesquisa em vários trabalhos (A. Vaccaro, 2009)
A maioria dos trabalhos apresentados na literatura sobre fluxo de carga considerando incertezas é baseada em algoritmos como desacoplado-rápido e Newton-Raphson, que são adequados para sistemas de transmissão, mas muitas vezes não convergem para sistemas de distribuição devido a sua configuração radial e uma alta relação R/X (Kersting, 2013), além do flat start desses sistemas. Dessa forma, métodos de varredura direto/reversa foram desenvolvidos especificamente para fluxo de carga em redes de distribuição (Das et al, 1994). Em Das (2002) é apresentado um fluxo de carga baseado em aritmética intervalar considerando o método de varredura soma de corrente. Em Gu et al (2014) é apresentado um fluxo de carga baseado em aritmética afim considerando o método de varredura soma de corrente.

Neste trabalho é apresentado um fluxo de carga baseado em aritmética intervalar considerando o método de varredura soma de potência. Nesta metodologia, as operações aritméticas ficam mais simples que no método da soma de corrente, pois não precisa trabalhar com aritmética intervalar complexa. 


\section{FORMULAÇÃO DO PROBLEMA}

\subsection{Aritmética Intervalar}

A aritmética intervalar é um modelo numérico baseado em faixas para computação numérica onde cada quantidade real é representada por um intervalo fechado de números. Os intervalos podem ser aplicados para representar valores desconhecidos, para controlar o erro de arredondamento, para representar dados inexatos, aproximações e erros de truncamento. A definição da aritmética intervalar é mostrada a seguir.

Considere que $\mathrm{X}=[\mathrm{x}, \mathrm{x}]$ e $\mathrm{Y}=[\mathrm{y}, \mathrm{y}]$ são dois intervalos. A adição, subtração, multiplicação, divisão e distância desses dois intervalos são definidas como,

$$
\begin{gathered}
\hat{X}+\hat{Y}=[\underline{x}+\underline{y}, \underline{x}+\underline{y}] \\
\widehat{\mathrm{X}}-\widehat{\mathrm{Y}}=[\underline{\mathrm{x}}-\underline{\mathrm{y}}, \underline{\mathrm{x}}-\underline{\mathrm{y}}] \\
\widehat{\mathrm{X}} * \widehat{\mathrm{Y}}=[\min (\underline{\mathrm{x}} * \underline{\mathrm{y}}, \underline{\mathrm{x}} * \underline{\mathrm{y}}, \underline{\mathrm{x}} * \underline{\mathrm{y}}, \underline{\mathrm{x}} * \underline{\mathrm{y}}), \\
\max (\underline{\mathrm{x}} * \underline{\mathrm{y}}, \underline{\mathrm{x}} * \underline{\mathrm{y}}, \underline{\mathrm{x}} * \underline{\mathrm{y}}, \underline{\mathrm{x}} * \underline{\mathrm{y}})] \\
\widehat{\mathrm{X}} \div \widehat{\mathrm{Y}}=\widehat{\mathrm{X}} * \widehat{\mathrm{Y}}-1
\end{gathered}
$$

Onde $\widehat{Y}-1=[1 / \underline{y}, 1 / \underline{y}]$ se $0 \notin=[\underline{y}$,

$$
\text { y] }
$$

$\operatorname{Dist}(\hat{X}, \hat{Y})=\max (|\underline{x}-\underline{y}|,|\underline{x}-\underline{y}|)$

\subsection{Fluxo de carga de soma de potência intervalar}

O fluxo de carga de soma de potência (Das et al, 1994) é utilizado tendo como entrada cargas e dados de linha definidos como intervalos da seguinte forma,

$$
\begin{aligned}
\hat{P} c_{i} & =P c_{i}{ }^{*}[1-\text { inc_P} / 100,1+\text { inc_P/100] } \\
\hat{Q} c_{i} & =Q c_{i}{ }^{*}[1 \text {-inc_Q/100,1+inc_Q/100] } \\
\hat{R}_{i j} & =R_{i j}{ }^{*}[1 \text {-inc_R/100,1+inc_R/100] } \\
\hat{X}_{i j} & =X_{i j}{ }^{*}[1 \text {-inc_X/100,1+inc_X/100] }
\end{aligned}
$$

onde $P c_{i}$ e $Q c i$, são a potência ativa e reativa base da barra i e $\hat{P} c_{i}$ e $\hat{Q} c_{i}$ são seus respectivos intervalos com incertezas de inc_P e inc_Q. $R_{i j}$ e $X_{i j}$, são as resistências e reatâncias base do trecho i-j e $\hat{R}_{i j}$ e $\hat{X}_{i j}$ são seus respectivos intervalos com incertezas de inc_R e inc_X. Os valores bases dessas variáveis são os valores determinados pelos transformadores ( $\mathrm{P} \mathrm{e} \mathrm{Q}$ ) e pelos cabos ( $\mathrm{R}$ e X).

Reescrevendo as equações do método da soma de potência (Das et al, 1994) em termos de intervalos, temos,

$$
\begin{gathered}
\hat{A}=\hat{P}_{i} * \hat{R}_{i j}+\widehat{Q}_{i} * \hat{X}_{i j}-0.5 * \hat{P}_{i} *\left|\widehat{V}_{l}\right|^{2} \\
\hat{B}=\left(\hat{R}_{i j}{ }^{2}+\hat{X}_{i j}{ }^{2}\right)^{*}\left(\hat{P}_{i}{ }^{2}+\hat{Q}_{i}{ }^{2}\right) \\
\hat{C}=\sqrt{\hat{A}^{2}-\hat{B}}
\end{gathered}
$$

$$
\widehat{V}_{j}=\sqrt{\hat{C}-\hat{A}}
$$

O fluxo de carga de Soma de Potência Intervalar (SPI) compreende os seguintes passos:

1. Inicialmente, considerar nulas as perdas ativas e reativas no alimentador;

2. Definir os intervalos das variáveis de entrada baseado nas equações de (7) a (10);

3. Calcular os fluxos intervalares de P e Q para cada barra considerando a configuração do alimentador. O fluxo intervalar na barra $i$ é definido como a soma das cargas intervalares e das perdas intervalares das barras e trechos que estão a sua jusante acrescido da sua própria carga intervalar;

4. Calcular os vetores auxiliares A e B empregando as equações (11) e (12), respectivamente;

5. Calcular o vetor auxiliar $\mathrm{C}$ empregando a equação (13);

6. Calcular a tensão do final do trecho empregando a equação (14).

7. Repetir os passos 2 a 6 para todos os trechos do alimentador.

8. Repetir os passos 2 a 7 enquanto houver variação significativa na distância (6) entre os intervalos da tensão do passo anterior e o atual.

\section{ESTUDO DE CASO}

Para ilustrar a aplicação do algoritmo proposto são escolhidos quatro alimentadores radiais de $30,68,282$ e 358 barras. Os dados dos alimentadores de 30 e 68 barras são encontrados em Das, B, (2002). Os dados do alimentador de 282 barras são encontrados em Alves e Sousa, (2014) e os dados do alimentador de 358 barras são mostrados no final do artigo (Apêndice A e B). Todos esses dados representam um ponto de operação do sistema e serão considerados como os valores base para definição das incertezas. A solução de fluxo de potência obtida por uma simulação de Monte Carlo não sequencial (SMC) é usada como referência. Foram gerados 1.000 .000 de amostras formadas por valores aleatórios da demanda de carga e dos dados de linha dentro de suas faixas de incertezas.

Foram consideradas incertezas nas demandas de $\pm 2 \%, \pm 5 \%$, $\pm 10 \%, \pm 15 \%$ e $\pm 20 \%$. Em todos os casos foi considerada uma incerteza de $\pm 2 \%$ nos dados de linha. As figuras de 1 a 4 apresentam os erros relativos das soluções do algoritmo proposto e o SMC (tomado como referência). Os erros são apresentados para os limites inferiores e superiores das soluções intervalares encontradas para cada sistema de teste com as imprecisões consideradas. A tabela 1 mostra o máximo erro encontrado para cada sistema de teste. Os limites obtidos no SMC para todos os sistemas de teste encontram-se no interior das faixas aritméticas obtidas. Seus intervalos são mais estreitos, contudo, não são significativas as diferenças obtidas (Tabela 1). 

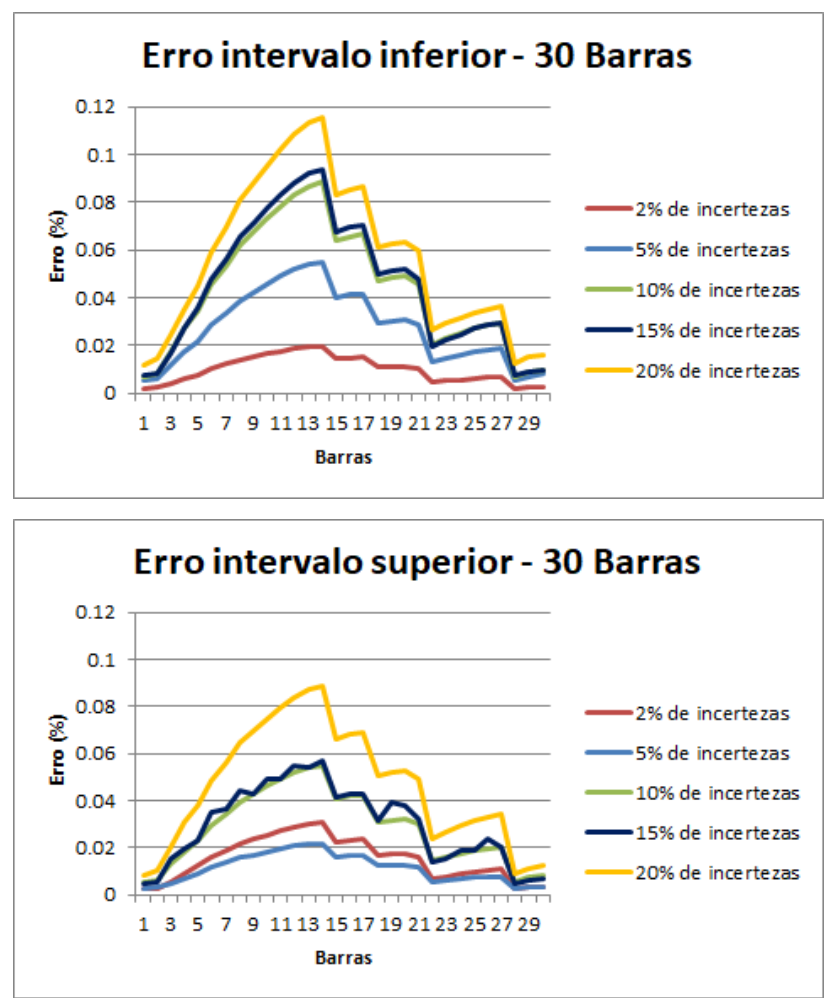

Figura 1. Erro relativo do intervalo inferior e superior das tensões obtido pelo SPI e SMC para o alimentador de 30 barras.
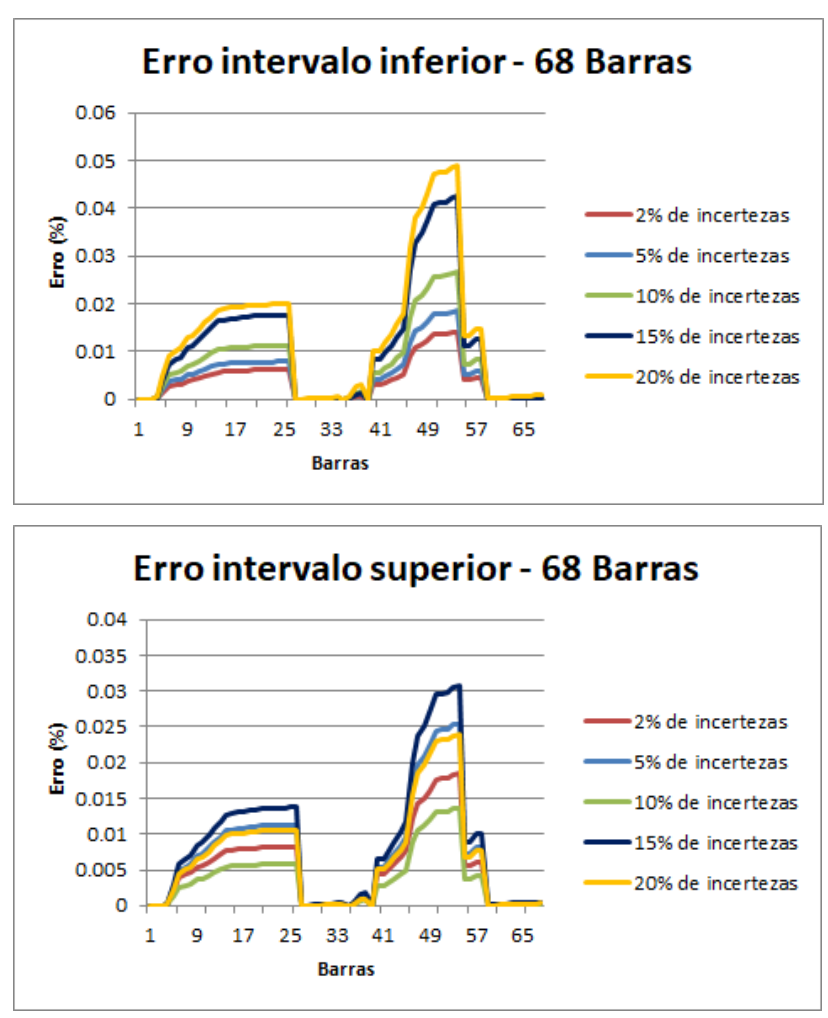

Figura 2. Erro relativo do intervalo inferior e superior das tensões obtido pelo SPI e SMC para o alimentador de 68 barras.
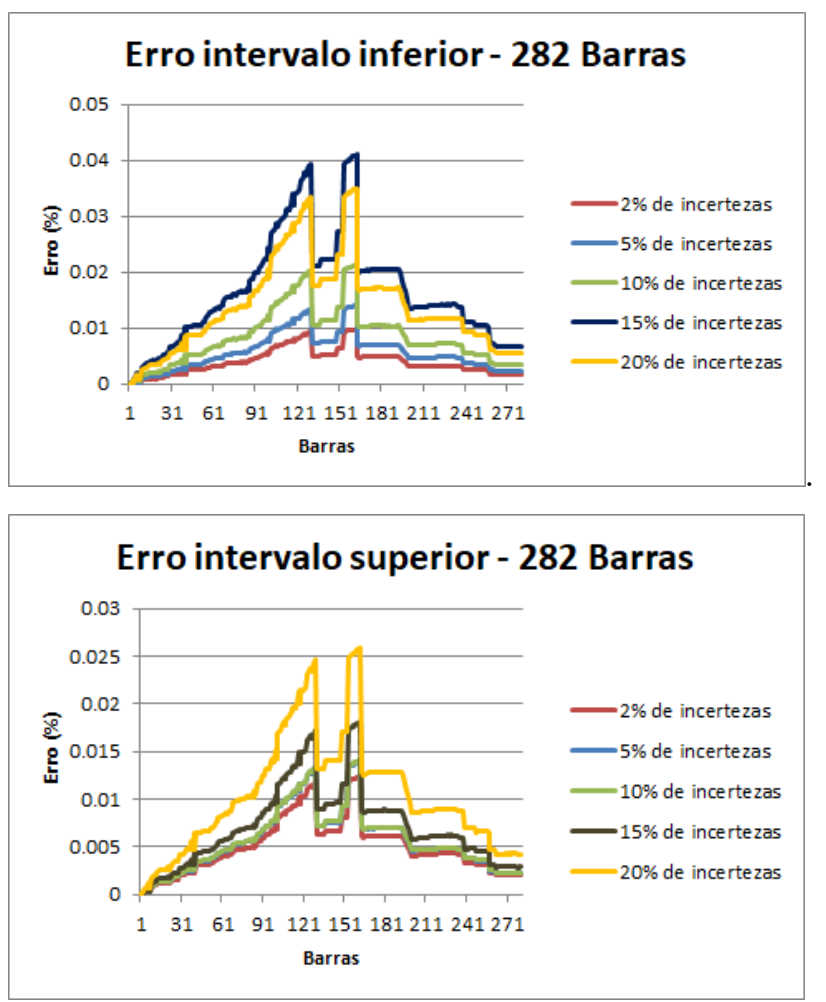

Figura 3. Erro relativo do intervalo inferior e superior das tensões obtido pelo SPI e SMC para o alimentador de 282 barras.

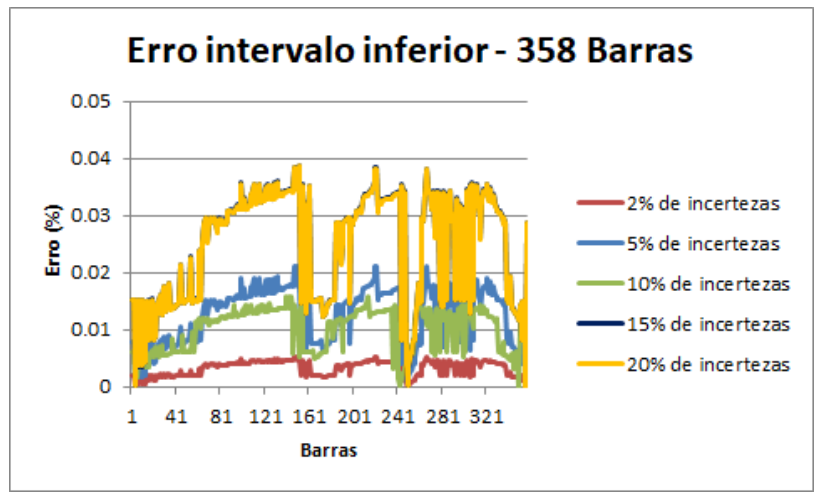

\section{Erro intervalo superior - 358 Barras}

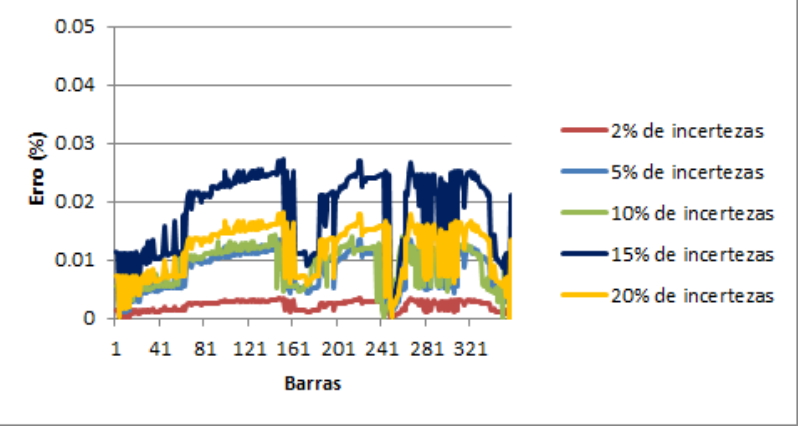


Figura 4. Erro relativo do intervalo superior das tensões obtido pelo SPI e SMC para o alimentador de 358 barras.

Tabela 1. Máximo erro relativo entre as soluções obtidas pelo SPI e SMC para cada sistema de teste.

\begin{tabular}{|c|c|}
\hline Alimentador & Máximo erro (\%) \\
\hline 30 & 0.1183 \\
\hline 68 & 0.0491 \\
\hline 282 & 0.0410 \\
\hline 358 & 0.0388 \\
\hline
\end{tabular}

A Tabela 2 apresenta os valores médios do número de iterações e do tempo computacional necessário à convergência do SPI. O fluxo de potência intervalar baseado no método de soma de corrente descrito em Das (2002) foi usado para comparação. Os mesmos parâmetros para teste de convergência foram utilizados em ambos os algoritmos, bem como, as incertezas de demanda e de parâmetros de linha. A formulação proposta se mostrou a mais eficiente tanto no tempo computacional como pelo número de iterações.

Tabela 2. Valores médios do número de iterações e do tempo computacional necessário à convergência do algoritmo proposto.

\begin{tabular}{|c|l|c|c|}
\hline \multirow{2}{*}{ Alimentador } & & SPI & $\begin{array}{c}\text { Das } \\
(2002)\end{array}$ \\
\hline \multirow{2}{*}{30} & Iterações & 4 & 9 \\
\cline { 2 - 4 } & Tempo(s) & 0.0065 & 0.0244 \\
\hline \multirow{2}{*}{68} & Iterações & 4 & 5 \\
\cline { 2 - 4 } & Tempo(s) & 0.0148 & 0.0301 \\
\hline \multirow{2}{*}{282} & Iterações & 4 & 5 \\
\cline { 2 - 4 } & Tempo(s) & 0.0608 & 0.1231 \\
\hline \multirow{2}{*}{358} & Iterações & 3 & 4 \\
\cline { 2 - 4 } & Tempo(s) & 0.058 & 0.1265 \\
\hline
\end{tabular}

\section{CONCLUSÕES}

Neste trabalho foi apresentado uma metodologia para o tratamento de incertezas no problema de fluxo de potência aplicada em redes de distribuição, onde as variáveis de entrada e de saída são modeladas por intervalos. O fluxo de potência com incertezas é uma ferramenta muito útil nas situações onde seriam necessárias diversas simulações do problema de fluxo de potência convencional. Para efeito de comparação e validação dos resultados são realizadas simulações de Monte Carlo de diversos casos do problema de fluxo de potência convencional.

Os resultados obtidos são excelentes visto que o maior erro encontrado foi de $0.2 \%$. Comparando os métodos de varredura intervalar de soma de corrente e soma de potência, o método proposto se mostrou maior eficiência computacional.

A ferramenta desenvolvida mostra grande potencial para uso em sistemas reais, pois seus resultados se aproximam dos casos extremos de operação. Como próximo passo nesta pesquisa, pretende-se incluir geração distribuída no fluxo de carga intervalar.

\section{AGRADECIMENTOS}

Os autores agradecem ao IFMA (Instituto Federal de Educação, Ciência e Tecnologia do Maranhão), e à Fundação de Amparo à Pesquisa e Desenvolvimento Científico do Maranhão (FAPEMA) pelo apoio ao desenvolvimento do projeto.

\section{REFERÊNCIAS}

A. Vaccaro and D. Villacci, Radial power flow tolerance analysis by interval constraint propagation, IEEE Trans. Power Syst., vol. 24, no. 1, pp. 28-39, Feb. 2009

Alves, H. N. and Sousa, R. S. (2014). A multi-population genetic algorithm to solve multi-objective remote switches allocation problem in distribution networks. In 2014 IEEE symposium on proceedings of computational intelligence for engineering solutions (CIES) (pp. 155164), 9-12 December 2014.

Bijwe, P.R., Hanmandlu, M. and Pande, V. N. (2005). Fuzzy power flow solutions with reactive limits and multiple uncertainties. Electric Power Systems Research, vol. 76, pp. $145-152$.

Borkowska, B. (1974). Probabilistic Load Flow. IEEE Transactions on Power Apparatus and Systems, vol. PAS-93, no. 6, pp. 752-755.

Das, B. (2002) Radial distribution system power flow using interval arithmetic. Int $J$ Electr Power Energy Syst. 24(10):827-36.

Das, D.; Nagi, H.S. and Kothari, D.P. (1994). Novel method for solving radial distribution net-work. IEE Proceedings Generation, Transmission and Distribution, Vol. 141, no. 4, pp. 291-298.

Gu W., Luo L., Ding T., Meng X. and W. Sheng. (2014). An affine arithmetic-based algorithm for radial distribution system power flow with uncertainties. Int. J. Elect. Power Energy Syst., vol.58, pp.242-245.

Kenarangui, R. and Seifi, A. (1994). Fuzzy Power Flow Analysis. Electric Power Systems Re-search, vol. 29, pp. 105-109.

Kersting, W. H. (2013). Distribution System Mod-eling and Analysis. 3.ed. Boca Ranton: CRC Pres.

Li, W. (2005). Risk Assessment of Power Systems: Models, Methods, and Applications. New York: IEEE Press/Wiley.

Pereira, L.E.S., Da Costa V.M. and Rosa A.L.S. (2012). Interval arithmetic in current injection power flow analysis. Int J Electr Power Energy Syst;43(1):1106-13. 
Su, C. L. (2005). Probabilistic load flow computation using point estimate method. IEEE Trans-actions on Power Systems, vol. 20, pp. 1843-1851.

X. Liao, K. Liu, Y. Zhang, K. Wang, L. Qin (2017), Interval method for uncertain power flow analysis based on Taylor inclusion function. IET Generation, Transmission \& Distribution, vol. 11, no.5, pp. 1270-1278.

Zhang, C. H. Chen, H. Hgan, P. Yang, and D. Hua. (2017). A mixed interval power flow analysis under rectangular and polar coordinate sys-tem. IEEE Trans. Power Syst., vol. 32 , no. 2 , pp. 1422-1429. 
Apêndice A. ALIMENTADOR DE 358 BARRAS (Sec - Seção, C - Cabo, L - Comprimento (M), S - Potência Complexa - KVA)

\begin{tabular}{|c|c|c|c|c|c|c|c|c|c|c|c|c|c|c|c|c|c|c|c|}
\hline Sec & $\mathrm{C}$ & $\mathrm{L}$ & $\mathrm{S}$ & $\mathrm{Sec}$ & $\mathrm{C}$ & $\mathrm{L}$ & $\mathrm{S}$ & $\mathrm{Sec}$ & $\mathrm{C}$ & $\mathrm{L}$ & $\mathrm{Sec}$ & $\mathrm{S}$ & $\mathrm{C}$ & $\mathrm{L}$ & $\mathrm{S}$ & $\mathrm{Sec}$ & $\mathrm{C}$ & $\mathrm{L}$ & $\mathrm{S}$ \\
\hline $0-1$ & 1 & 1 & 0 & $66-73$ & 2 & 65 & 0 & $140-145$ & 2 & 53 & 112.5 & 211-217 & 2 & 166 & 45 & $274-289$ & 2 & 348 & 45 \\
\hline $1-2$ & 1 & 32 & 0 & $66-74$ & 2 & 39 & 75 & $136-146$ & 2 & 55 & 150 & $209-218$ & 2 & 135 & 75 & $275-290$ & 2 & 26 & 75 \\
\hline $2-3$ & 1 & 10 & 0 & $74-75$ & 2 & 210 & 0 & $\begin{array}{l}136-147 \\
\end{array}$ & 2 & 541 & 15 & $212-219$ & 2 & 35 & 75 & $283-291$ & 2 & 122 & 0 \\
\hline $3-4$ & 1 & 345 & 0 & $69-76$ & 3 & 69 & 150 & $142-148$ & 2 & 24 & 0 & $215-220$ & 2 & 155 & 0 & $283-292$ & 2 & 87 & 0 \\
\hline $4-5$ & 1 & 74 & 45 & $71-77$ & 2 & 40 & 0 & $142-149$ & 2 & 35 & 0 & $213-221$ & 1 & 89 & 0 & $284-293$ & 2 & 189 & 5 \\
\hline $4-6$ & 2 & 9 & 0 & $72-78$ & 2 & 94 & 0 & $143-150$ & 2 & 32 & 0 & $213-222$ & 2 & 8 & 0 & $285-294$ & 2 & 33 & 0 \\
\hline $5-7$ & 1 & 79 & 0 & $70-79$ & 1 & 77 & 75 & 143-151 & 1 & 24 & 75 & $214-223$ & 1 & 253 & 0 & $286-295$ & 2 & 149 & 0 \\
\hline $6-8$ & 2 & 222 & 0 & $70-80$ & 2 & 163 & 0 & $144-152$ & 2 & 151 & 0 & $216-224$ & 2 & 140 & 75 & $287-296$ & 2 & 89 & 0 \\
\hline $7-9$ & 1 & 73 & 0 & 69-81 & 2 & 113 & 30 & $144-153$ & 2 & 54 & 75 & $214-225$ & 2 & 50 & 75 & $293-297$ & 2 & 121 & 0 \\
\hline $8-10$ & 2 & 64 & 45 & $73-82$ & 2 & 122 & 75 & $145-154$ & 2 & 143 & 0 & $217-226$ & 2 & 117 & 0 & $294-298$ & 2 & 212 & 0 \\
\hline $9-11$ & 1 & 90 & 0 & $77-83$ & 2 & 46 & 0 & $142-155$ & 2 & 54 & 75 & $223-227$ & 1 & 103 & 0 & 292-299 & 2 & 136 & 75 \\
\hline $11-12$ & 1 & 185 & 0 & $79-84$ & 1 & 235 & 0 & $148-156$ & 2 & 94 & 0 & $220-228$ & 2 & 240 & 0 & $295-300$ & 2 & 98 & 0 \\
\hline $12-13$ & 1 & 62 & 75 & $76-85$ & 3 & 84 & 112.5 & $151-157$ & 1 & 54 & 0 & $220-229$ & 2 & 7 & 0 & $291-301$ & 2 & 101 & 30 \\
\hline $12-14$ & 2 & 26 & 0 & $75-86$ & 2 & 132 & 0 & $153-158$ & 2 & 233 & 0 & $221-230$ & 1 & 179 & 75 & $296-302$ & 2 & 72 & 45 \\
\hline $14-15$ & 2 & 123 & 0 & $78-87$ & 2 & 79 & 75 & 154-159 & 2 & 77 & 0 & $222-231$ & 2 & 188 & 0 & $297-303$ & 2 & 105 & 0 \\
\hline $13-16$ & 1 & 170 & 0 & 75-88 & 2 & 39 & 75 & $149-160$ & 2 & 34 & 112.5 & $230-232$ & 2 & 63 & 0 & $297-304$ & 2 & 70 & 0 \\
\hline $16-17$ & 1 & 139 & 0 & $80-89$ & 2 & 87 & 45 & 150-161 & 2 & 81 & 112.5 & $228-233$ & 2 & 58 & 0 & 299-305 & 2 & 375 & 0 \\
\hline $15-18$ & 2 & 112 & 0 & $85-90$ & 3 & 49 & 225 & $148-162$ & 2 & 18 & 112.5 & $229-234$ & 2 & 151 & 75 & $298-306$ & 2 & 89 & 0 \\
\hline $15-19$ & 2 & 59 & 45 & $83-91$ & 3 & 65 & 0 & $152-163$ & 2 & 39 & 75 & $231-235$ & 2 & 52 & 0 & $298-307$ & 2 & 29 & 0 \\
\hline $17-20$ & 1 & 74 & 0 & $83-92$ & 3 & 256 & 0 & $158-164$ & 2 & 122 & 0 & $227-236$ & 2 & 9 & 0 & $300-308$ & 2 & 76 & 45 \\
\hline $18-21$ & 2 & 49 & 75 & $84-93$ & 1 & 99 & 0 & $156-165$ & 2 & 11 & 0 & $227-237$ & 2 & 37 & 0 & $305-309$ & 2 & 118 & 0 \\
\hline $20-22$ & 3 & 71 & 0 & $84-94$ & 2 & 34 & 75 & $160-166$ & 2 & 132 & 150 & $234-238$ & 2 & 14 & 0 & $303-310$ & 2 & 70 & 0 \\
\hline $20-23$ & 1 & 43 & 0 & $86-95$ & 3 & 40 & 75 & 161-167 & 2 & 46 & 0 & $233-239$ & 2 & 142 & 0 & $304-311$ & 2 & 8 & 0 \\
\hline $22-24$ & 3 & 65 & 0 & $90-96$ & 2 & 87 & 75 & $157-168$ & 2 & 21 & 0 & $232-240$ & 2 & 36 & 0 & $304-312$ & 2 & 39 & 0 \\
\hline $23-25$ & 1 & 53 & 75 & $91-97$ & 2 & 162 & 0 & $157-169$ & 2 & 30 & 0 & $232-241$ & 2 & 145 & 0 & $308-313$ & 2 & 4 & 0 \\
\hline $25-26$ & 1 & 171 & 0 & $92-98$ & 2 & 261 & 30 & 159-170 & 2 & 113 & 0 & $232-242$ & 2 & 25 & 0 & $303-314$ & 2 & 53 & 150 \\
\hline $24-27$ & 3 & 28 & 0 & $93-99$ & 1 & 62 & 0 & $159-171$ & 2 & 39 & 0 & $236-243$ & 2 & 169 & 0 & $306-315$ & 2 & 97 & 75 \\
\hline $24-28$ & 2 & 57 & 112.5 & $94-100$ & 2 & 172 & 0 & $156-172$ & 2 & 41 & 112.5 & $237-244$ & 2 & 42 & 45 & $307-316$ & 2 & 474 & 0 \\
\hline $24-29$ & 2 & 35 & 0 & 96-101 & 3 & 20 & 0 & $156-173$ & 2 & 237 & 112.5 & $235-245$ & 2 & 92 & 112.5 & $312-317$ & 2 & 97 & 0 \\
\hline $27-30$ & 3 & 28 & 0 & $97-102$ & 2 & 163 & 0 & $158-174$ & 2 & 106 & 45 & $240-246$ & 2 & 76 & 0 & $313-318$ & 2 & 40 & 0 \\
\hline $26-31$ & 3 & 22 & 0 & 98-103 & 2 & 470 & 0 & 164-175 & 2 & 162 & 0 & $239-247$ & 2 & 46 & 0 & $309-319$ & 2 & 122 & 0 \\
\hline $26-32$ & 1 & 20 & 0 & $99-104$ & 1 & 136 & 0 & $\begin{array}{ll}170-176 \\
\end{array}$ & 2 & 18 & 0 & $241-248$ & 2 & 154 & 75 & $310-320$ & 2 & 201 & 75 \\
\hline $30-33$ & 3 & 38 & 0 & $100-105$ & 3 & 191 & 75 & 166-177 & 2 & 31 & 0 & $242-249$ & 2 & 83 & 0 & $311-321$ & 2 & 493 & 75 \\
\hline $31-34$ & 3 & 157 & 75 & $101-106$ & 3 & 56 & 0 & $167-178$ & 2 & 45 & 0 & $243-250$ & 2 & 172 & 0 & $319-322$ & 2 & 230 & 0 \\
\hline $32-35$ & 1 & 156 & 45 & 103-107 & 2 & 28 & 0 & $\begin{array}{l}168-179 \\
\end{array}$ & 1 & 34 & 0 & $244-251$ & 2 & 123 & 0 & $320-323$ & 2 & 168 & 75 \\
\hline $34-36$ & 3 & 63 & 0 & $102-108$ & 2 & 34 & 0 & $\begin{array}{l}169-180 \\
\end{array}$ & 2 & 187 & 75 & $239-252$ & 2 & 145 & 45 & $321-324$ & 2 & 320 & 0 \\
\hline $33-37$ & 3 & 95 & 75 & 104-109 & 1 & 39 & 45 & 164-181 & 2 & 199 & 0 & 238253 & 2 & 141 & 45 & $317-325$ & 2 & 29 & 0 \\
\hline $35-38$ & 1 & 172 & 45 & $\begin{array}{l}109-110 \\
\end{array}$ & 1 & 219 & 0 & $171-182$ & 2 & 26 & 75 & $238-254$ & 2 & 199 & 45 & $318-326$ & 2 & 13 & 112.5 \\
\hline $38-39$ & 1 & 242 & 0 & 106-111 & 2 & 88 & 75 & 165-183 & 2 & 24 & 112.5 & $238-255$ & 2 & 22 & 0 & $318-327$ & 2 & 34 & 30 \\
\hline $37-40$ & 3 & 60 & 0 & 106-112 & 3 & 33 & 0 & 165-184 & 2 & 118 & 75 & $248-256$ & 2 & 44 & 0 & $317-328$ & 2 & 34 & 75 \\
\hline $36-41$ & 3 & 95 & 45 & $108-113$ & 2 & 150 & 45 & 164-185 & 2 & 20 & 75 & $249-257$ & 2 & 148 & 0 & $327-329$ & 2 & 40 & 0 \\
\hline $36-42$ & 2 & 25 & 0 & $106-114$ & 2 & 41 & 0 & $181-186$ & 2 & 36 & 0 & $250-258$ & 2 & 145 & 0 & $322-330$ & 2 & 159 & 225 \\
\hline $41-43$ & 3 & 173 & 0 & $107-115$ & 2 & 56 & 30 & $178-187$ & 2 & 134 & 0 & $247-259$ & 2 & 76 & 112.5 & $323-331$ & 2 & 492 & 45 \\
\hline $39-44$ & 1 & 98 & 0 & $107-116$ & 2 & 98 & 0 & $182-188$ & 2 & 56 & 0 & $246-260$ & 2 & 208 & 45 & $325-332$ & 2 & 415 & 0 \\
\hline $40-45$ & 3 & 74 & 0 & 111-117 & 2 & 162 & 45 & $\begin{array}{l}179-189 \\
\end{array}$ & 1 & 231 & 112.5 & $251-261$ & 2 & 86 & 0 & $324-333$ & 2 & 144 & 0 \\
\hline $42-46$ & 2 & 171 & 112.5 & $112-118$ & 2 & 65 & 225 & $180-190$ & 2 & 161 & 150 & $246-262$ & 2 & 103 & 75 & $326-334$ & 2 & 301 & 0 \\
\hline $39-47$ & 2 & 85 & 75 & 113-119 & 2 & 267 & 0 & $177-191$ & 2 & 39 & 45 & $259-263$ & 2 & 77 & 0 & $331-335$ & 2 & 724 & 10 \\
\hline $39-48$ & 2 & 88 & 112.5 & $110-120$ & 1 & 61 & 112.5 & $175-192$ & 2 & 93 & 75 & $261-264$ & 2 & 17 & 0 & $329-336$ & 2 & 16 & 0 \\
\hline $45-49$ & 3 & 44 & 0 & $110-121$ & 3 & 83 & 0 & $175-193$ & 2 & 11 & 0 & $260-265$ & 2 & 146 & 0 & $329-337$ & 2 & 131 & 0 \\
\hline $43-50$ & 3 & 58 & 0 & $110-122$ & 2 & 211 & 75 & $175-194$ & 2 & 24 & 0 & $256-266$ & 2 & 33 & 0 & $330-338$ & 2 & 76 & 75 \\
\hline $43-51$ & 2 & 30 & 0 & $118-123$ & 2 & 57 & 0 & $176-195$ & 2 & 126 & 0 & $256-267$ & 2 & 66 & 0 & $332-339$ & 2 & 171 & 30 \\
\hline $44-52$ & 1 & 227 & 0 & $120-124$ & 1 & 123 & 0 & $176-196$ & 2 & 79 & 75 & $256-268$ & 2 & 151 & 75 & $336-340$ & 2 & 130 & 0 \\
\hline $44-53$ & 2 & 42 & 45 & $119-125$ & 2 & 400 & 30 & $189-197$ & 1 & 263 & 0 & $257-269$ & 2 & 126 & 0 & $337-341$ & 2 & 215 & 0 \\
\hline 50-54 & 3 & 58 & 0 & $121-126$ & 2 & 28 & 0 & $187-198$ & 2 & 45 & 75 & $257-270$ & 2 & 173 & 75 & $335-342$ & 2 & 336 & 0 \\
\hline $49-55$ & 3 & 37 & 75 & $117-127$ & 2 & 179 & 0 & $190-199$ & 2 & 34 & 0 & $258-271$ & 2 & 269 & 112.5 & $340-343$ & 2 & 144 & 15 \\
\hline $49-56$ & 2 & 18 & 0 & $126-128$ & 2 & 44 & 0 & $186-200$ & 2 & 30 & 0 & $258-272$ & 2 & 64 & 75 & $341-344$ & 2 & 42 & 0 \\
\hline $52-57$ & 1 & 327 & 75 & 123-129 & 2 & 36 & 300 & $186-201$ & 2 & 21 & 0 & $263-273$ & 2 & 30 & 0 & $343-345$ & 2 & 265 & 0 \\
\hline $49-58$ & 2 & 120 & 150 & 123-130 & 2 & 46 & 0 & $187-202$ & 2 & 105 & 75 & $263-274$ & 2 & 8 & 0 & $344-346$ & 2 & 191 & 0 \\
\hline $51-59$ & 2 & 92 & 500 & $125-131$ & 2 & 53 & 25 & $186-203$ & 2 & 79 & 150 & $265-275$ & 2 & 34 & 0 & $345-347$ & 2 & 1703 & 0 \\
\hline $55-60$ & 3 & 33 & 0 & 124-132 & 1 & 80 & 0 & $188-204$ & 2 & 81 & 75 & $267-276$ & 2 & 308 & 0 & $346-348$ & 2 & 494 & 5 \\
\hline $54-61$ & 2 & 41 & 112.5 & $123-133$ & 2 & 225 & 112.5 & $188-205$ & 2 & 117 & 0 & $268-277$ & 2 & 220 & 0 & $345-349$ & 2 & 436 & 15 \\
\hline 54-62 & 2 & 52 & 0 & 124-134 & 2 & 11 & 0 & $198-206$ & 2 & 70 & 75 & $270-278$ & 2 & 84 & 0 & $346-350$ & 2 & 70 & 75 \\
\hline $57-63$ & 1 & 40 & 0 & 124-135 & 2 & 56 & 75 & $197-207$ & 1 & 102 & 75 & $264-279$ & 2 & 45 & 45 & $347-351$ & 2 & 152 & 0 \\
\hline $56-64$ & 2 & 104 & 45 & $131-136$ & 2 & 127 & 0 & $197-208$ & 2 & 8 & 0 & $264-280$ & 2 & 76 & 0 & $348-352$ & 2 & 735 & 75 \\
\hline $61-65$ & 2 & 235 & 0 & 130-137 & 2 & 42 & 112.5 & $197-209$ & 2 & 12 & 0 & $266-281$ & 2 & 126 & 75 & $347-353$ & 2 & 42 & 75 \\
\hline $62-66$ & 2 & 75 & 0 & $132-138$ & 1 & 131 & 0 & 199-210 & 2 & 110 & 0 & $269-282$ & 2 & 125 & 45 & $351-354$ & 2 & 42 & 0 \\
\hline 60-67 & 3 & 79 & 0 & 128-139 & 2 & 39 & 0 & $200-211$ & 2 & 60 & 112.5 & $276-283$ & 2 & 183 & 0 & $351-355$ & 2 & 12 & 0 \\
\hline 63-68 & 1 & 203 & 75 & $128-140$ & 2 & 27 & 0 & $201-212$ & 2 & 123 & 0 & $277-284$ & 2 & 25 & 0 & $352-356$ & 2 & 29 & 0 \\
\hline $67-69$ & 3 & 1 & 0 & $129-141$ & 2 & 58 & 75 & $207-213$ & 1 & 201 & 0 & $278-285$ & 2 & 127 & 0 & $354-357$ & 2 & 484 & 500 \\
\hline $68-70$ & 1 & 173 & 0 & $137-142$ & 2 & 47 & 0 & $210-214$ & 2 & 301 & 0 & $279-286$ & 2 & 76 & 0 & $355-358$ & 2 & 526 & 75 \\
\hline $65-71$ & 2 & 29 & 0 & $138-143$ & 1 & 70 & 0 & $206-215$ & 2 & 63 & 75 & $280-287$ & 2 & 152 & 112.5 & & & & \\
\hline $65-72$ & 2 & 28 & 0 & $139-144$ & 2 & 344 & 0 & $208-216$ & 2 & 61 & 0 & $273-288$ & 2 & 10 & 0 & & & & \\
\hline
\end{tabular}

Apêndice B. IMPEDÂNCIA DOS CABOS DO ALIMENTADOR DE 358 BARRAS

\begin{tabular}{|c|c|c|c|}
\hline Cabo & 1 & 2 & 3 \\
\hline $\mathrm{R}(\Omega / \mathrm{km})$ & 0.6047 & 2.4301 & 0.9633 \\
\hline $\mathrm{X}(\Omega / \mathrm{km})$ & 0.4483 & 0.5022 & 0.4673 \\
\hline
\end{tabular}


